\title{
Legal Sources
}

Asylum Act, (AsylA), of 26 June 1998 (Status as of 1 January 2018), 142.31. https://www.admin.ch/opc/en/classified-compilation/19995092/index. html, last accessed 05.04.2018.

Asylverordnung 1 über Verfahrensfragen, (Asylverordnung 1, AsylV 1), vom 11. August 1999 (Stand am 1. März 2017). 142.311. https://www.admin.ch/opc/ de/classified-compilation/19994776/index.html, last accessed 05.04.2018. BVGE 2015/3, Bundesverwaltungsgericht, Grundsatzurteil. Begründung der Flüchtlingseigenschaft durch Wehrdienstverweigerung oder Desertion. Analyse der diesbezüglichen Lage in Syrien. https://entscheide.weblaw. ch/cache.php?link=BVGE-2015-3, last accessed 05.04.2018.

BVGer D-5577/2013, Bundesverwaltungsgericht, Urteil vom 9. Juli 2014. http://links.weblaw.ch/d/BVGer-D-5577/2013, last accessed 05.04.2018.

BVGer E-3361/2014, Bundesverwaltungsgericht, Urteil vom 6. Mai 2015. http://links.weblaw.ch/BVGer-E-3361/2014, last accessed 05.04.2018.

Charter of Fundamental Rights of the European Union. 2012/C 326/02, http:// eur-lex.europa.eu/legal-content/EN/TXT/HTML/?uri=CELEX:12012P/ TXT\&from=EN, last accessed 05.04.2018.

Convention Relating to the Status of Refugees (adopted on 28 July 1951) - the "1951 Refugee Convention" - and Protocol Relating to the Status of Refugees (adopted on 16 December 1967), http://www.unhcr.org/3b66c2aa10, last accessed 05.04.2018.

Court of Justice of the European Union, Judgement C-199-12, 7 November 2013, curia.europa.eu, last accessed 05.04.2018.

ECHR Affaire N.A. c. SUISSE (Requête no 50364/14), Arrêt, Strassbourg, 30 Mai 2017. http://hudoc.echr.coe.int/eng?i=001-173790 last accessed 05.06.2020.

EMARK 2006/3, http://www.ark-cra.ch/emark/2006/03.htm, last accessed 05.04.2018. 
EMARK 2006/18, http://www.ark-cra.ch/emark/2006/18.htm, last accessed 05.04.2018.

European Convention on Human Rights, as amended by Protocols Nos. 11 and 14, supplemented by Protocols Nos. 1, 4, 6, 7, 12 and 13. https://www. echr.coe.int/Pages/home.aspx?p=basictexts\&c, last accessed 05.04.2018.

Federal Act on Administrative Procedure, (Administrative Procedure Act APA), of 20 December 1968 (Status as of 1 January 2018). 172.021. https:// www.admin.ch/opc/en/classified-compilation/19680294/index.html, last accessed 05.04.2018.

Federal Act on Foreign Nationals, (Foreign Nationals Act FNA), of 16 December 2005 (Status as of 1 January 2018). 142.20. https://www.admin.ch/opc/ en/classified-compilation/20020232/index.html, last accessed 05.04.2018.

Federal Constitution of the Swiss Confederation, of 18 April 1999 (Status as of 12 February 2017). 101. https://www.admin.ch/opc/en/classified-com pilation/19995395/index.html, last accessed 05.04.2018.

Regulation (EU) No 604/2013, https://eur-lex.europa.eu/legal-content/EN/T $\mathrm{XT/HTML/?uri=CELEX:32013R0604 \& from=de,} \mathrm{last} \mathrm{accessed} \mathrm{05.04.2018.}$ 


\section{Social Sciences}
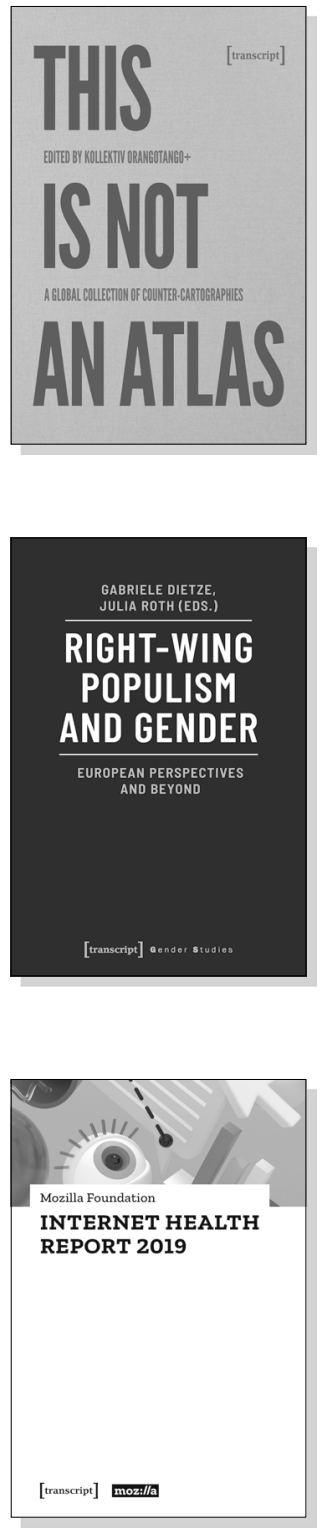

kollektiv orangotango + (ed.)

This Is Not an Atlas

A Global Collection of Counter-Cartographies

2018, 352 p., hardcover, col. ill.

34,99€ (DE), 978-3-8376-4519-4

E-Book: free available, ISBN 978-3-8394-4519-8

Gabriele Dietze, Julia Roth (eds.)

\section{Right-Wing Populism and Gender}

European Perspectives and Beyond

April 2020, 286 p., pb., ill.

$35,00 €$ (DE), 978-3-8376-4980-2

E-Book: 34,99 $€$ (DE), ISBN 978-3-8394-4980-6

Mozilla Foundation

Internet Health Report 2019

2019, 118 p., pb., ill.

19,99 € (DE), 978-3-8376-4946-8

E-Book: free available, ISBN 978-3-8394-4946-2 


\section{Social Sciences}

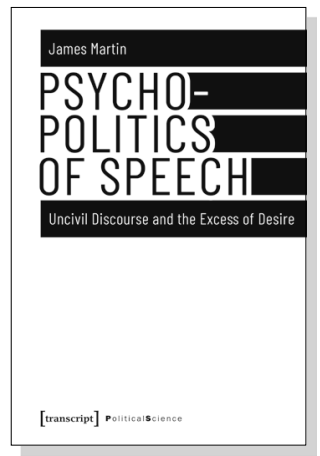

James Martin

\section{Psychopolitics of Speech}

Uncivil Discourse and the Excess of Desire

2019, 186 p., hardcover

79,99€ (DE), 978-3-8376-3919-3

E-Book:

PDF: 79,99 € (DE), ISBN 978-3-8394-3919-7

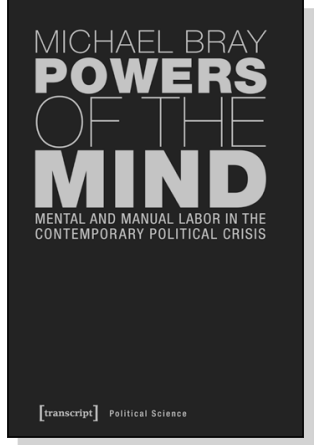

Michael Bray

\section{Powers of the Mind}

Mental and Manual Labor

in the Contemporary Political Crisis

2019, 208 p., hardcover

99,99€ (DE), 978-3-8376-4147-9

E-Book:

PDF: 99,99€ (DE), ISBN 978-3-8394-4147-3

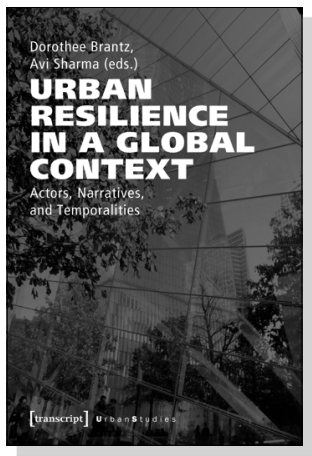

Dorothee Brantz, Avi Sharma (eds.)

\section{Urban Resilience in a Global Context}

Actors, Narratives, and Temporalities

October 2020, 224 p., pb.

30,00 € (DE), 978-3-8376-5018-1

E-Book: available as free open access publication PDF: ISBN 978-3-8394-5018-5 